Regularized phase tracking technique for demodulation of isochromatics from a single tricolour image

This article has been downloaded from IOPscience. Please scroll down to see the full text article.

2002 Meas. Sci. Technol. 13132

(http://iopscience.iop.org/0957-0233/13/1/317)

View the table of contents for this issue, or go to the journal homepage for more

Download details:

IP Address: 147.96.14.16

The article was downloaded on 14/05/2013 at 17:03

Please note that terms and conditions apply. 


\title{
Regularized phase tracking technique for demodulation of isochromatics from a single tricolour image
}

\author{
J A Quiroga ${ }^{1}$, M Servin ${ }^{2}$ and J L Marroquin ${ }^{3}$ \\ ${ }^{1}$ Departamento de Óptica, Facultad de Ciencias Físicas, Universidad Complutense, Ciudad \\ Universitaria s/n, 28040 Madrid, Spain \\ ${ }^{2}$ Centro de Investigaciones en Optica AC, PO Box 1-948, 37150 Leon Gto, México \\ ${ }^{3}$ Centro de Investigación en Matematicas, PO Box 402, Guanajuato, Gto 36240, México \\ E-mail: aq@ fis.ucm.es
}

Received 12 July 2001, accepted for publication 8 November 2001

Published 12 December 2001

Online at stacks.iop.org/MST/13/132

\begin{abstract}
In this work we propose a robust fringe demodulation technique applied to the analysis of tricolour isochromatic fringe patterns produced in photoelasticity. The method used is a regularized phase tracking (RPT) algorithm, which takes into account the different information contained in the three bands of the colour image obtained with an RGB-CCD camera.

Automatic determination of the zero-order isochromatic and the area of interest, the use of a discrete fluorescent illumination and the use of the RPT configures a robust method for automatic demodulation of isochromatic fringe patterns. The performance of the method is discussed and experimental results are presented.
\end{abstract}

Keywords: stress, retardation, photoelasticity, polariscope, fringe pattern processing

\section{Introduction}

During recent years a big effort has been made to develop automatic methods for the analysis of photoelastic fringe patterns. For this purpose, different techniques such as phase shifting [1], Fourier transform [2], spectral content analysis [3], tricolour image analysis [4], RGB calibration [5], load stepping [6] and regularization techniques [7] have been applied. One of the objectives of all these techniques is the analysis of photoelastic fringe patterns by using the smallest number of images.

In the case of isochromatic fringe patterns, the tricolour, spectral content analysis, RGB calibration, regularization and Fourier transform techniques need only one image, which is obviously the smallest possible number. This is an interesting feature in applications where temporal varying phenomena are to be analysed such as photoviscoelasticity or flow analysis.

The spectral content analysis technique [3] determines the isochromatic fringe order by using a white light source and measuring the output spectrum emerging from a polariscope. This technique is very accurate but the measurement is point- wise, and a spectrometer is needed to perform the analysis. Related to the spectral content analysis technique, the RGB calibration method [5] is based on the construction of an RGB look-up table that gives the relation between a given RGB triplet and the fractional isochromatic fringe order. In this case the experimental set-up is simple: a polariscope and a colour CCD camera. The main problem is that using conventional illumination and eight-bit digitization for each RGB channel this technique can discriminate only up to four to five orders due to the lack of contrast in the colour signal as the fringe order increases. In the tricolour image analysis [4], a plane polariscope set-up is used and RGB images are acquired. In the case of isochromatics, by splitting the three RGB channels and combining them a nonlinear equation is set from which the fractional isochromatic order can be extracted point by point. Due to the plane polariscope configuration used, isoclinics produce low-modulation areas in which the nonlinear equation cannot be solved, thus making some kind of interpolation necessary.

A totally different approach is used by the Fourier transform technique [2]: here monochromatic illumination is 
used and a carrier is introduced by means of a quartz wedge; further Fourier transform analysis computes the isochromatic phase. The advantages of the Fourier transform technique are well known; however, near the stress concentration areas, the gradients of the isochromatic phase (and thus the spatial frequency content) are high, making the application of the Fourier analysis difficult.

Another possibility for the analysis of isochromatic fringe patterns is the regularization techniques [8]. In these algorithms the modulating phase of a fringe pattern is demodulated by minimizing a suitable cost function consisting of a fidelity term and a regularization term. The fidelity term reflects the proximity of the obtained phase to the observed data. The regularization term imposes a priori knowledge about the obtained phase, usually piecewise continuity. Recently, Servin and Quiroga [9] have applied a modified regularization technique for the analysis of monochrome isochromatic fringe patterns. However, the technique has limitations in the case of fringe patterns with high dynamic range of spatial frequencies and saddle points, which are usual cases in photoelastic fringe patterns.

In this work we present a regularization algorithm for the demodulation of the phase from a single isochromatic RGB image obtained with a standard RGB-CCD camera. In this case, the multiplicative relationship between the modulating phases of each RGB fringe pattern is used. This algorithm is complemented with an RGB-value comparison method used to determine a point or set of points with known isochromatic order. Finally discrete fluorescent illumination instead of conventional incandescent lamps is used to increase the maximum discernible isochromatic fringe order.

This work is organized as follows. In section 2 the regularized phase tracking technique for RGB fringe patterns is presented. Section 3 discusses the utilization of discrete fluorescent illumination. In section 4 we show how from the RGB values of the image a point on the zero-order or the first-order isochromatic fringe can be found. Finally, experimental results and comparison with a standard phase sampling algorithm are presented in section 5 .

\section{The RGB regularized phase tracking algorithm}

\subsection{The regularized phase tracking technique}

The standard mathematical model for a fringe pattern may be written as

$$
I(x, y)=a(x, y)+b(x, y) \cos [\phi(x, y)]
$$

where $a(x, y)$ and $b(x, y)$ are the background illumination and the amplitude modulation respectively. Finally $\phi(x, y)$ is the modulating phase that we want to recover from the fringe pattern.

The basic idea of the regularized phase tracking (RPT) technique [10] is that locally the intensity of the fringe pattern may be considered as spatially monochromatic, so its irradiance may be modelled as a sinusoidal function phase modulated by a plane $p(\cdot)$. Thus by minimizing an appropriate cost function that takes into account the differences between the measured irradiance and the proposed model it will be possible to demodulate the phase $\phi(x, y)$ from the irradiance
$I(x, y)$. In the standard RPT the cost function to be minimized at each site $(x, y)$ is

$$
\begin{gathered}
U(x, y)=\sum_{(\xi, \eta) \in\left(N_{x, y} \cap S\right)}\left\{\left[I^{\prime}(\xi, \eta)-\cos p(x, y, \xi, \eta)\right]^{2}\right. \\
\left.+\beta\left[\phi_{0}(\xi, \eta)-p(x, y, \xi, \eta)\right]^{2} m(\xi, \eta)\right\}
\end{gathered}
$$

with

$p(x, y, \xi, \eta)=\phi_{0}(x, y)+\omega_{x}(x, y)(x-\xi)+\omega_{y}(x, y)(y-\eta)$

where $S$ denotes the region of interest (ROI) where valid data are present, $N_{x, y}$ is the neighbourhood region around the site $(x, y)$ where the phase is being estimated and $m(x, y)$ is an indicator field which is equal to one if the phase at site $(x, y)$ has already been estimated, and zero otherwise. The fringe pattern $I^{\prime}(x, y)$ is the high-pass filtered and amplitudenormalized version of $I(x, y)$; this operation is performed in order to eliminate the low-frequency background $a(x, y)$ and to make $b(x, y) \approx 1$. The fields $\omega_{x}(x, y)$ and $\omega_{y}(x, y)$ are the estimated local frequencies along the $x$ and $y$ directions respectively; they also represent the slopes of the locally fitted phase plane. Finally the parameter $\beta$ is a constant denominated regularization parameter which controls (along with the size of $\left.N_{x, y}\right)$ the smoothness of the detected phase $\phi_{0}(x, y)$.

The first term in (2) attempts to keep the local fringe model close to the observed irradiance in a least-squares sense within the neighbourhood $N_{x, y}$. This term is not sensitive to sudden phase $2 \pi$ jumps because the same result is obtained for the $\cos ()$ function. To avoid this problem, the second term enforces the smoothness and continuity by comparing the phase predicted by the plane $p(x, y, \xi, \eta)$ with the phase of the previously detected pixels, $\phi_{0}(\xi, \eta)$, marked by $m(\xi, \eta)$. In this way a large value for $\beta$ will produce very smooth results and a small value can give discontinuous results. Typical values for $\beta$ are in the range of one to ten.

To demodulate the phase from a given fringe pattern with the RPT method we need to minimize the cost function $U(x, y)$ at each point with respect to the variables $\phi_{0}(x, y), \omega_{x}(x, y)$ and $\omega_{y}(x, y)$. From equation (3) this is is a nonlinear problem, so fast quadratic optimization techniques cannot be applied. A common method used to minimize $U(x, y)$ is the fixed-step gradient descent. That is, given $\boldsymbol{r}(x, y)=\left(\phi_{0}, \omega_{x}, \omega_{y}\right)^{T}$ we estimate the phase and frequency components at each point according to the recursion

$$
r^{k+1}=r^{k}-\mu \nabla_{r} U(x, y)
$$

where $\mu$ is the step of descent of the gradient search. The symbol $\nabla_{r}$ denotes the gradient of $U(x, y)$ with respect to the three components of $\boldsymbol{r}$.

The gradient descent method needs a starting condition $\boldsymbol{r}^{0}$ in (4). In the case of isochromatic demodulation this means that for the initial pixel (seed pixel) at $\left(x_{0}, y_{0}\right)$ inside $S$, the isochromatic order must be known (the way we select the starting point with known isochromatic order will be shown in section 4). When the seed vector $\boldsymbol{r}\left(x_{0}, y_{0}\right)$ is optimized, the regularizing term of (2) is not taken into account given that the seed pixel is the first one that is demodulated and, as a consequence, the indicator function $m(x, y)$ is equal to zero over $S$. Once the seed vector $\boldsymbol{r}\left(x_{0}, y_{0}\right)$ is estimated we may proceed with the rest of the sites in $S$. The RPT must 
follow a continuous sequential path starting at the seed pixel, propagating the estimated seed vector, $\boldsymbol{r}\left(x_{0}, y_{0}\right)$, as the initial condition for the following adjacent site. So, in this way, the RPT proceeds from pixel to pixel following a given path.

As mentioned, the RPT demodulates sequentially the fringe pattern, starting at one seed pixel and then propagating from it until the whole ROI has been processed. The way this sequential scanning is performed has been demonstrated to be a key point in the demodulation process by means of the RPT (actually for any sequential image processing technique). The best choice we have found is the use of a quality-map guided scanning strategy. Ströbel [11] first proposed this in the context of phase unwrapping. The objective of the quality map is to guide the RPT in such a way that it processes locally in the first place the highest-quality areas, leaving aside the low-quality regions so that they are processed at the end. In this way the possible errors that occur in the areas of low quality will be confined to these areas.

A good quality map can be obtained from the modulation term $b(x, y)$ of equation (1). One can easily obtain this modulation if the Fourier transform or phase shifting techniques are used; for example in [12] and [13] this modulation information is used successfully in combination with the RPT. However, in the case of using only one fringe pattern this modulation information is, in general, difficult to obtain. Another possibility to use as the quality map is the fringe pattern itself, that is following as far as possible the isophase lines; this strategy has been used successfully in [9] and [14] in the demodulation of isochromatic and speckle fringe patterns respectively.

\subsection{Demodulation of $R G B$ isochromatic fringe patterns}

For the isochromatic calculation we used a diffuselight circular polariscope with monochromatic ( $\mathrm{Na}$ lamp, $\lambda_{\mathrm{Na}}=589 \mathrm{~nm}$ ) and discrete fluorescent illumination in the circular dark field (CDF) configuration [15]. The quarterwave plates of the polariscope are matched for $\lambda_{\mathrm{Na}}$. Using the monochromatic source the output is a monochrome image given by

$$
I_{\mathrm{Na}}=\frac{I_{0 \mathrm{Na}}}{2}\left(1-\cos \left(\delta_{\mathrm{Na}}\right)\right)
$$

where $I_{0 \mathrm{Na}}$ is the incident illumination and $\delta_{\mathrm{Na}}$ is the isochromatic phase for $\lambda_{\mathrm{Na}}$, that under elastic conditions is given by

$$
\delta_{\mathrm{Na}}=2 \pi \frac{C_{\mathrm{Na}}}{\lambda_{\mathrm{Na}}}\left(\sigma_{1}-\sigma_{2}\right)
$$

where $C_{\mathrm{Na}}$ is the photoelastic constant of the material for $\lambda_{\mathrm{Na}}$ and $\sigma_{1}$ and $\sigma_{2}$ are the two principal stresses generated by the corresponding state of stress. If we apply the RPT demodulation scheme to this case the parameters $\phi_{0}, \omega_{x}$ and $\omega_{y}$ of equation (3) will be identified as $\delta_{\mathrm{Na}}, \partial \delta_{\mathrm{Na}} / \partial x$ and $\partial \delta_{\mathrm{Na}} / \partial y$ respectively.

If discrete fluorescent illumination is used and the image is captured with an RGB-CCD camera the acquired image has three bands, namely R, G and B, whose irradiance images for the CDF configuration are
Table 1. Experimentally obtained values for the $K_{i j}$.

\begin{tabular}{lllll}
\hline & \multicolumn{4}{c}{$i$} \\
\cline { 2 - 5 }$j$ & $\mathrm{Na}$ & $\mathrm{R}$ & $\mathrm{G}$ & $\mathrm{B}$ \\
\hline $\mathrm{Na}$ & 1 & 0.961 & 1.098 & 1.493 \\
$\mathrm{R}$ & 1.040 & 1 & 1.144 & 1.536 \\
$\mathrm{G}$ & 0.911 & 0.874 & 1 & 1.394 \\
$\mathrm{~B}$ & 0.670 & 0.651 & 0.717 & 1 \\
\hline
\end{tabular}

$$
\begin{aligned}
& I_{\mathrm{R}}=\frac{I_{0 R}}{2}\left(1-\cos \left(\delta_{\mathrm{R}}\right)\right) \\
& I_{\mathrm{G}}=\frac{I_{0 G}}{2}\left(1-\cos \left(\delta_{\mathrm{G}}\right)\right) \\
& I_{\mathrm{B}}=\frac{I_{0 B}}{2}\left(1-\cos \left(\delta_{\mathrm{B}}\right)\right)
\end{aligned}
$$

where the $I_{0 i}$ are the incident illuminations associated with each band. Due to the spectral response of each RGB channel and the discrete nature of the fluorescent illumination we can consider that the irradiance images (7) have a monochromatic character, which implies that the retardations can be described by

$$
\begin{aligned}
& \delta_{\mathrm{R}}=2 \pi \frac{C_{\mathrm{R}}}{\lambda_{\mathrm{R}}}\left(\sigma_{1}-\sigma_{2}\right) \\
& \delta_{\mathrm{G}}=2 \pi \frac{C_{\mathrm{G}}}{\lambda_{\mathrm{G}}}\left(\sigma_{1}-\sigma_{2}\right) \\
& \delta_{\mathrm{B}}=2 \pi \frac{C_{\mathrm{B}}}{\lambda_{\mathrm{B}}}\left(\sigma_{1}-\sigma_{2}\right)
\end{aligned}
$$

where $\mathrm{C}_{i}$ are the photoelastic constants for each one of the wavelengths $\lambda_{i}$. If we define $K_{i}=C_{i} / \lambda_{i}$, equations (6) and (8) indicate that by knowing one of the phases $\delta_{i}$, and knowing the ratios $K_{i, j}=K_{i} / K_{j}$, it is possible to compute the remaining retardations. For example, if we know $K_{\mathrm{Na}, \mathrm{R}}=K_{\mathrm{Na}} / K_{\mathrm{R}}$ (from specifications or experimentally), the retardation for the $\mathrm{Na}$ line can be recovered from the $\mathrm{R}$ channel one by $\delta_{\mathrm{Na}}=K_{\mathrm{Na}, \mathrm{R}} \delta_{\mathrm{R}}$. In our experiment, we have computed the ratios $K_{i, j}$ by calculating the retardation using the method described in [16] for each RGB channel for a diametrically loaded disc. The corresponding values are shown in table 1 .

As an example, figure 1 shows the $\mathrm{R}, \mathrm{G}$ and $\mathrm{B}$ bands of isochromatic CDF irradiance images corresponding to the bottom part of a diametrically loaded disc; the size of these images is $325 \times 768$ pixels.

To adapt the RPT to the case of RGB isochromatic fringe patterns the cost function must take into account the information contained in the three RGB bands; in particular this change will affect the fidelity term of the RPT whereas the regularization term will be unchanged. Taking this into account, the proposed cost function is

$$
\begin{aligned}
& U(x, y)=\sum_{(\xi, \eta) \in\left(N_{x, y} \cap S\right)}\left\{\left[I_{\mathrm{R}}^{\prime}(\xi, \eta)-\cos p_{\mathrm{R}}(x, y, \xi, \eta)\right]^{2}\right. \\
& +\left[I_{\mathrm{G}}^{\prime}(\xi, \eta)-\cos K_{\mathrm{G}, \mathrm{R}} p_{\mathrm{R}}(x, y, \xi, \eta)\right]^{2} \\
& +\cdots\left[I_{\mathrm{B}}^{\prime}(\xi, \eta)-\cos K_{\mathrm{B}, \mathrm{R}} p_{\mathrm{R}}(x, y, \xi, \eta)\right]^{2} \\
& \left.+\beta\left[\delta_{\mathrm{R}}(\xi, \eta)-p_{\mathrm{R}}(x, y, \xi, \eta)\right]^{2} m(\xi, \eta)\right\}
\end{aligned}
$$

with

$$
\begin{aligned}
& p_{\mathrm{R}}(x, y, \xi, \eta)=\delta_{\mathrm{R}}(x, y) \\
& \quad+\omega_{\mathrm{R} x}(x, y)(x-\xi)+\omega_{\mathrm{R} y}(x, y)(y-\eta)
\end{aligned}
$$



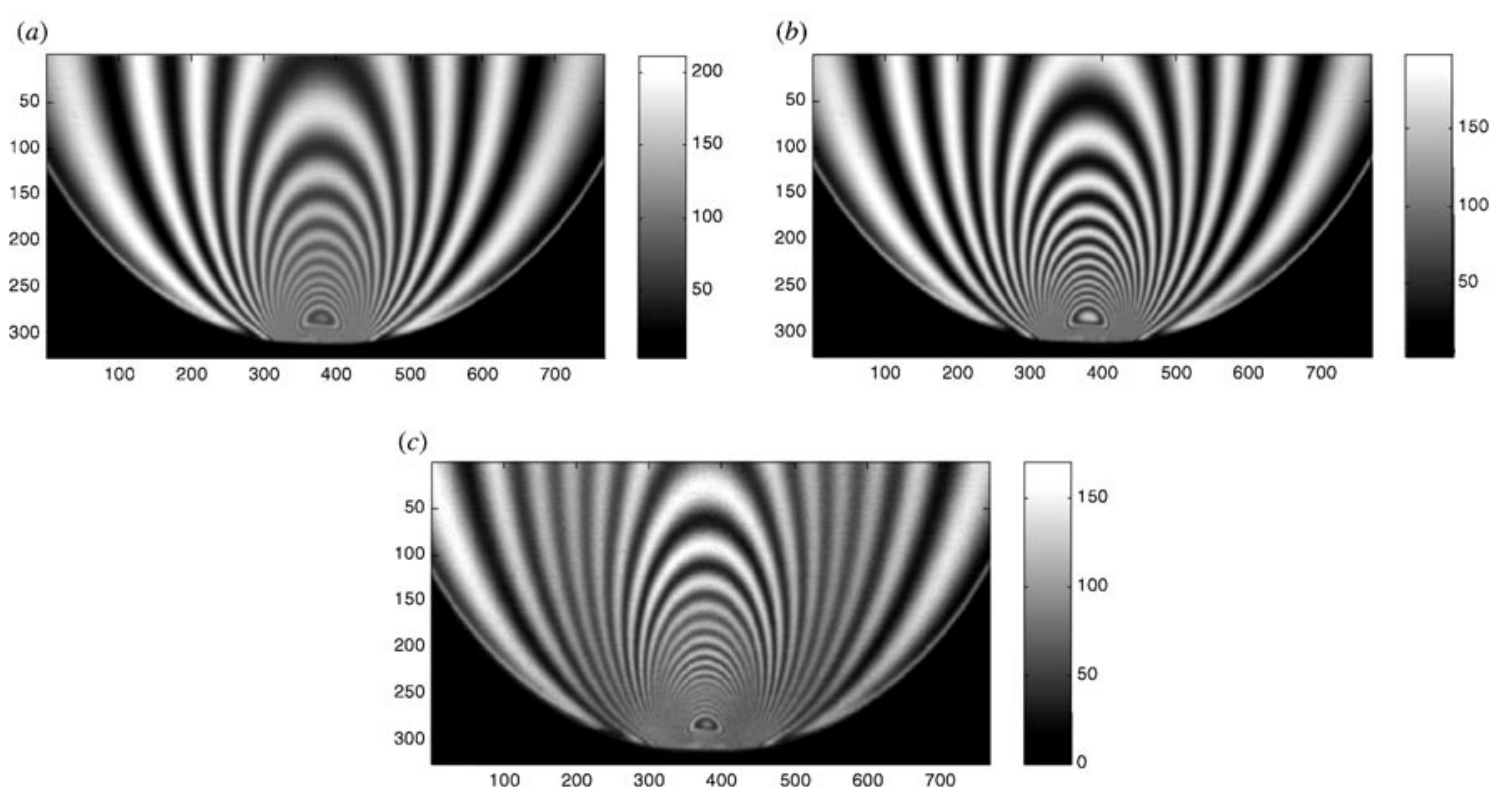

Figure 1. $(a),(b)$ and $(c)$ correspond to the R, G and B bands, respectively, of the isochromatic RGB pattern for a disc under diametrical compression obtained in the CDF configuration of a circular polariscope. The pictures show an enlarged portion of the bottom contact point.

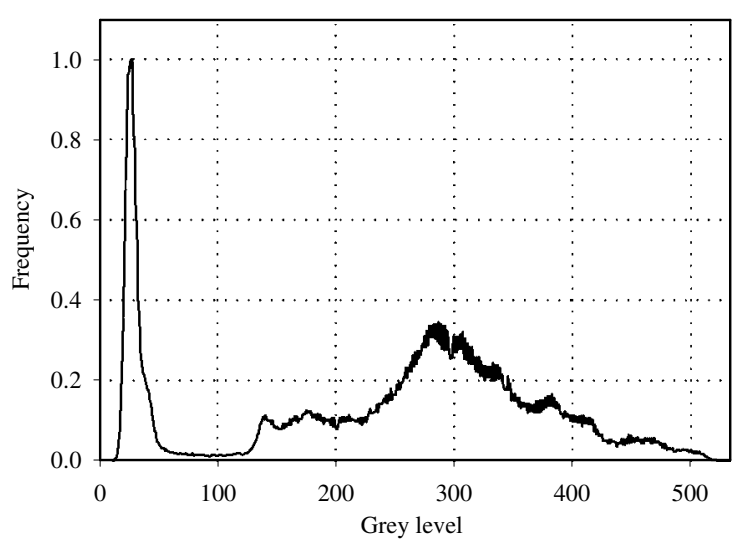

Figure 2. Histogram of the $I_{\mathrm{S}}$ image corresponding to figure 1. The two lobes correspond to the background (left) and to the fringe pattern (right).

where $I_{i}^{\prime}$ corresponds to the normalized irradiance of each one of the RGB channels, and $\omega_{\mathrm{R} x}$ and $\omega_{\mathrm{R} y}$ are the spatial frequencies associated with the $\delta_{\mathrm{R}}$ phase, these being the three parameters for which the cost function (9) is minimized point by point. Equations (9) and (10) means that with the RPT version for RGB images we demodulate the phase associated with one of the RGB bands (in our particular case the R channel) by using the multiplicative relationship between the phase of each band and given that the constants $K_{i, j}$ are known. As stated above, once $\delta_{\mathrm{R}}$ is computed the remaining retardations can be calculated by multiplying them by the appropriate constant.

In the case of RGB photoelasticity the ROI can be easily determined by thresholding the image resulting from computing the sum of the three bands of the RGB image

$$
I_{\mathrm{S}}=I_{\mathrm{R}}+I_{\mathrm{G}}+I_{\mathrm{B}}
$$

This image has a clear bimodal character in both circular dark field and circular bright field configurations of the

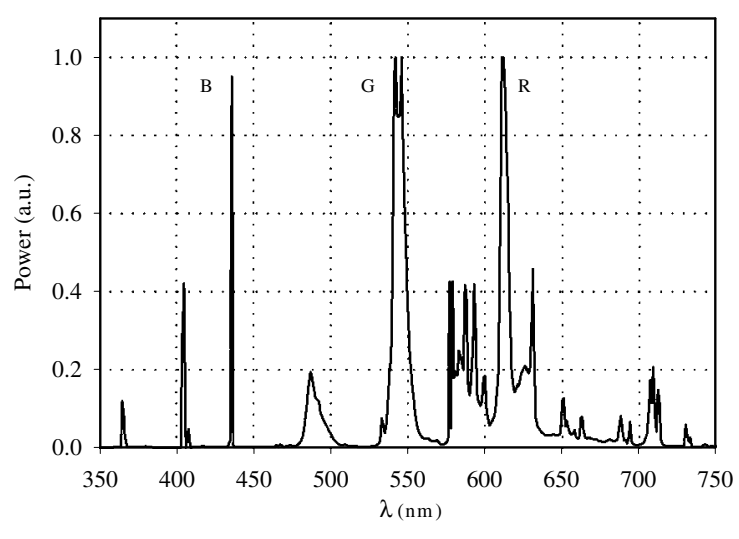

Figure 3. Spectrum of the fluorescent lamp used to obtain the images shown in this work.

polariscope. As an example figure 2 shows the histogram of $I_{\mathrm{S}}$ corresponding to the RGB image shown in figure 1. As can be seen, this histogram presents a clear bi-modal character, where the left lobe (lower values) corresponds to the background.

\section{Use of fluorescent illumination}

As a light source for photoelastic measurements, a wide number of different systems have been proposed. The classical incandescent lamp has been widely used but its limitations to produce sharp fringes with good modulation above isochromatic order four to five is well known. To overcome this problem several solutions have been proposed: a special illuminating tricolour source based on two discharge lamps and interference filters [4], CRT displays [17] and LEDbased systems [18]. In this work we propose the use of discrete fluorescent light source implemented as a commercial lowconsumption lamp, in order to overcome the above-mentioned problem of the modulation for high isochromatic orders. 

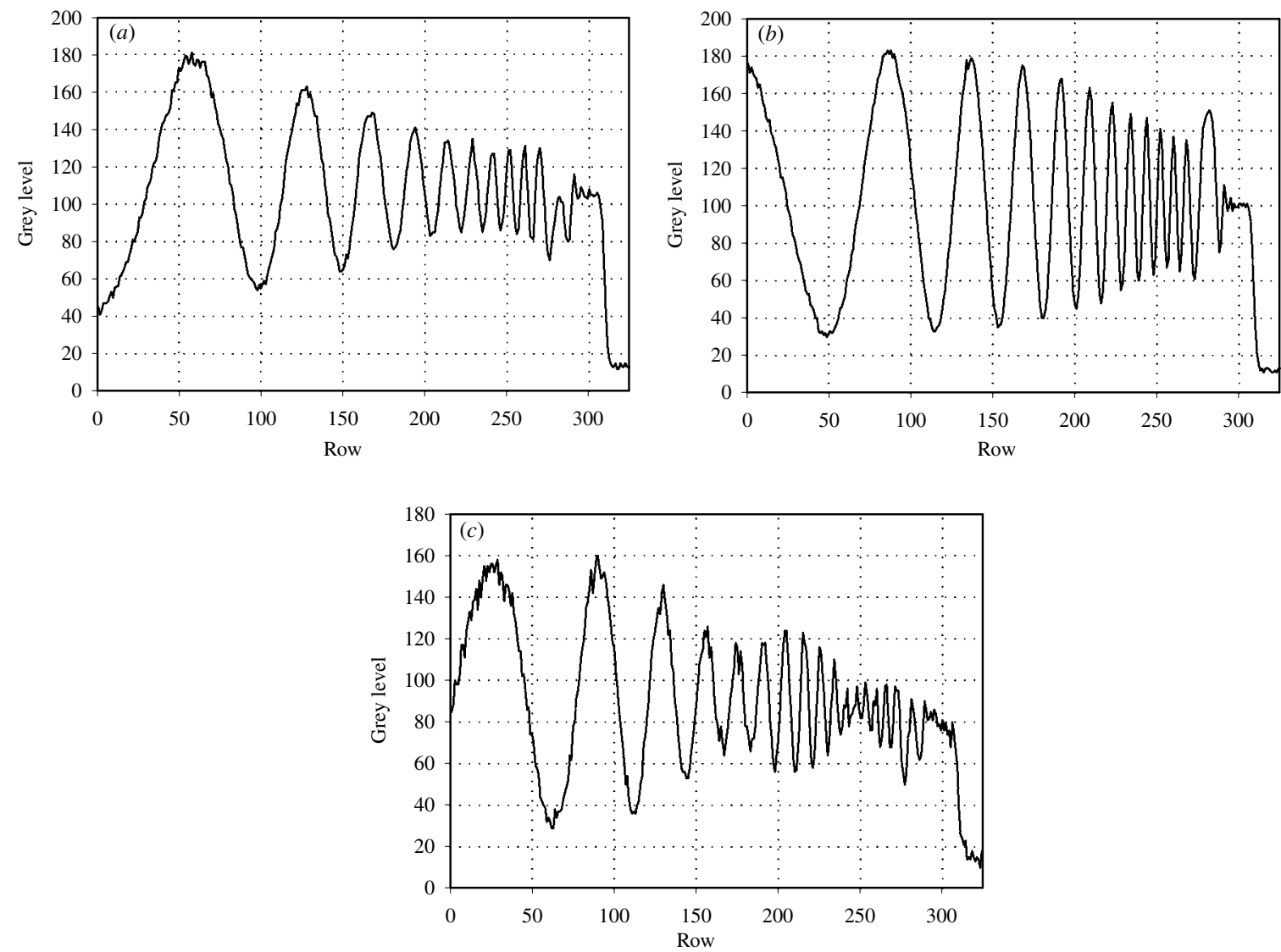

Figure 4. (a)-(c) Profile along central diameter (column 378) for the R, G and B bands respectively of figure 1.

The spectrum of the florescent lamps we used is shown in figure 3. As can be observed the red and green lines are highly monochromatic but the blue part consists mainly of two lines. In consequence, as can be seen in figure 1 , the $\mathrm{R}$ and $\mathrm{G}$ bands have sharp and well modulated fringes for high isochromatic orders, but in the case of the B channel it is possible to observe a beat in the fringe modulation due to the presence of two lines. Figure 4 shows the profiles of the fringe patterns depicted in figure 1 along the diameter of the disc (column 378). In the case of the $\mathrm{R}$ and $\mathrm{G}$ bands it is possible to observe the typical lack of modulation due to the stress gradients near the contact point. However in the case of the B band superposed on this lack of modulation the mentioned beat is observable.

For the RPT the modulation variation is undesirable because the cost function compares, in the fidelity term, the measured data with our model for the fringe pattern: a zerobackground and constant-modulation sinusoidal function. For this reason some processing of the acquired RGB irradiance images is necessary to eliminate the background and normalize the modulation.

The algorithm designed for the background elimination and modulation normalization is based on the use of two orthogonal bandpass filters [19]. The first filter is designed to remove the negative horizontal spatial frequencies, obtaining in this way an analytic signal, with phase $\varphi_{1}$, which will present discontinuities every time the local tangent of the fringes becomes horizontal. Thus the corresponding modulation, $m_{1}(x, y)$, will be very low at those points where the isophasic lines are horizontal. The second band pass filter is designed to remove the negative vertical spatial frequencies, obtaining a analytic signal, with phase $\varphi_{2}$, which will have discontinuities at those points where the isophasic lines have vertical tangent. Thus the modulation in this case, $m_{2}(x, y)$, will be very low at the points at which the isophasic lines are vertical.

If the fringe pattern is described by $I(x, y)=a(x, y)+$ $b(x, y) \cos (\varphi)$, we can use the phase maps, $\varphi_{1}$ and $\varphi_{2}$, to generate a normalized and background-corrected version of the fringe pattern given by

$$
\begin{aligned}
& I^{\prime}(x, y)=\cos (\varphi(x, y)) \\
& \quad \approx \frac{m_{1}(x, y) \cos \left(\varphi_{1}(x, y)\right)+m_{2} \cos \left(\varphi_{2}(x, y)\right)}{m_{1}(x, y)+m_{2}(x, y)} .
\end{aligned}
$$

In our experiments, the use of discrete fluorescent illumination and the normalization algorithm (12) permits us to have well modulated fringes for high isochromatic orders.

\section{Starting point selection}

As mentioned above the minimization of the RPT cost function (2) and its RGB version (9) is a nonlinear problem; additionally, it is a multimodal function. The meaning of this is that at the initial point, for which no extra information is available in its surroundings, many triplets $\left(\delta_{\mathrm{R}}, \omega_{\mathrm{R} x}, \omega_{\mathrm{R} y}\right)$ can be a good solution for the minimization of (9).

In the case of the gradient descent algorithm we use to solve the minimization of the cost function (9), it is enough for the initial pixel to give as starting value a triplet with 
$\omega_{\mathrm{R} x}=\omega_{\mathrm{R} y}=0$ and $\delta_{\mathrm{R}}=2 \pi M$, where $M$ is the order of the $\mathrm{R}$ isochromatic fringe pattern at the location of the starting point.

Thus, to ensure a correct starting of the RPT, a location with known fringe order must be found. With an a priori RGB calibration this can be easily accomplished but in general this is not possible due to the background and modulation change for different samples and experiments, so a 'blind' technique should be used.

Isotropic points are those for which the value of the difference between principal stresses is zero, that is $\sigma_{1}-\sigma_{2}=$ 0 ; in this case the isochromatic retardation $\delta$ is zero for all wavelengths. As a consequence isotropic points determine the location of the zero-order isochromatic fringe. In the CDF configuration according to (7) the three RGB intensities will have their minimum values at the zero-order fringe, thus its location can be easily found by finding the minimum of the $I_{\mathrm{S}}$ image given by (11). In this case $M=0$ and the starting triplet for the RPT algorithm will be $(0,0,0)$. However, the use of the zero-order fringe has some drawbacks. The first is that usually the zero-order fringe is located at one of the free borders of the object under study. This fact makes its detection unreliable because of the bad image quality in the vicinity of the border. In the second place, in the surroundings of $\delta_{\mathrm{R}}=0$ there is almost no phase difference between the three irradiances of the fidelity term of equation (9), therefore there is a lack of information in these places, making the starting of the RTP more unreliable with respect to the situation in which a certain amount of phase difference exists. Finally in the computation of the minimum of $I_{\mathrm{S}}$ problems can arise due to the non-uniformity of the incident illumination.

For these reasons we decide to look for a technique for the determination of a point with known order different from zero. The technique we propose is based on the computation of the $b$ RGB chromatic coordinate defined as

$$
b=\frac{I_{\mathrm{B}}}{I_{\mathrm{S}}}
$$

where $I_{\mathrm{S}}$ is given by (11). In general, the main advantage of using chromatic coordinates is that background variation and modulation effects are reduced.

We have found that the isochromatic first-order fringe for the $\mathrm{R}$ band can be located by finding the maximum of the chromatic co-ordinate $b$ inside the ROI. In this case $M=1$ and the starting triplet for the RTP is $(2 \pi, 0,0)$.

This procedure can be explained as follows. As we have said, when discrete spectrum fluorescent light is used, the three RGB bands can be considered monochromatic; that is, their intensity distributions can be described by (7). Thus we can rewrite (7) as

$$
\begin{gathered}
I_{\mathrm{R}}=\frac{I_{0 \mathrm{R}}}{2}\left(1-\cos \left(\delta_{\mathrm{R}}\right)\right) \\
I_{\mathrm{G}}=\frac{I_{0 \mathrm{G}}}{2}\left(1-\cos \left(K_{\mathrm{GR}} \delta_{\mathrm{R}}\right)\right) \\
I_{\mathrm{B}}=\frac{I_{0 \mathrm{~B}}}{2}\left(1-\cos \left(K_{\mathrm{BR}} \delta_{\mathrm{R}}\right)\right) .
\end{gathered}
$$

In our particular case (according to table 1) $K_{\mathrm{GR}}=1.144$ and $K_{\mathrm{BR}}=1.536$. If we represent the chromatic coordinate

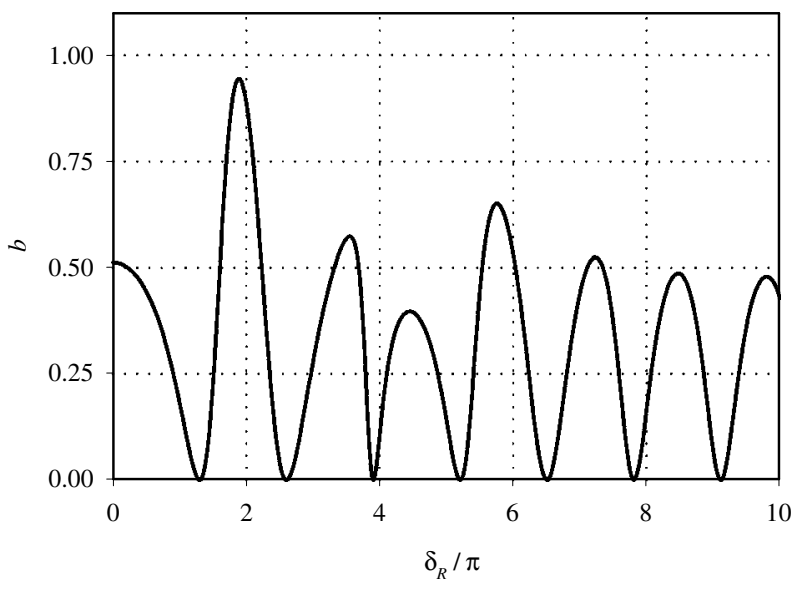

Figure 5. Chromatic coordinate $b$ as a function of $\delta_{\mathrm{R}}$ computed for the monochromatic case.

$b$ as a function of $\delta_{\mathrm{R}}$ for these particular values of $K_{\mathrm{GR}}$ and $K_{\mathrm{BR}}$ we shall observe a maximum located near $\delta_{\mathrm{R}} \approx 1.87 \pi$. Figure 5 shows the plot of this curve. In the monochromatic case this maximum is a global one in the range $0-10 \pi$ approximately, and this fact will limit the application of this method for isochromatic fringe patterns with no more than five orders. However the lack of monochromaticity (that appears even for the discrete spectrum fluorescent lamp) produces a lack of the modulation of the $b\left(\delta_{\mathrm{R}}\right)$ signal that makes this maximum global. We have tested this procedure to find the first-order $\mathrm{R}$ band isochromatic fringe with a discrete spectrum as well as continuous spectrum illuminating sources with different sample materials, always obtaining the correct result even up to order 16 for the $\mathrm{R}$ band. Additionally, numerical experiments performed with different combinations of illumination spectrum and camera responses have confirmed the method.

\section{Experimental results}

In our experiments we have used a diffuse light circular polariscope equipped with two illuminating sources: a monochrome Na lamp and discrete fluorescent lamps. For the image acquisition we used a three-chip RGB-CCD camera model JAI M90. The three-chip configuration guarantees the maximum possible spatial resolution.

As stated before, figure 1 shows the three RGB bands of an enlarged portion of a diametrically loaded disc. The size of the images is $325 \times 768$ pixels. Figure 6 shows the normalized irradiance for each one of the three bands shown in figure 1; these images correspond to the intensities $I_{i}^{\prime}(x, y)(i=\mathrm{R}, \mathrm{G}, \mathrm{B})$ of equation (9). In this figure the background suppression and modulation normalization are clearly observable even for the case of the B channel. Figure 7 shows the isochromatic phase $\delta_{\mathrm{R}}(x, y)$ obtained by the RTP that corresponds to the modulating phase of the $\mathrm{R}$ channel of figure 1. Although the RTP directly produces the unwrapped phase, this image is shown modulo $2 \pi$ for the sake of clarity. The RTP parameters used in this case were $N=9$ pixels (size of the neighbourhood) and $\beta=1$. Figure 8 shows the comparison for two profiles of the results obtained by the RTP and a phase shifting (PS) method [16] that uses eight images. 

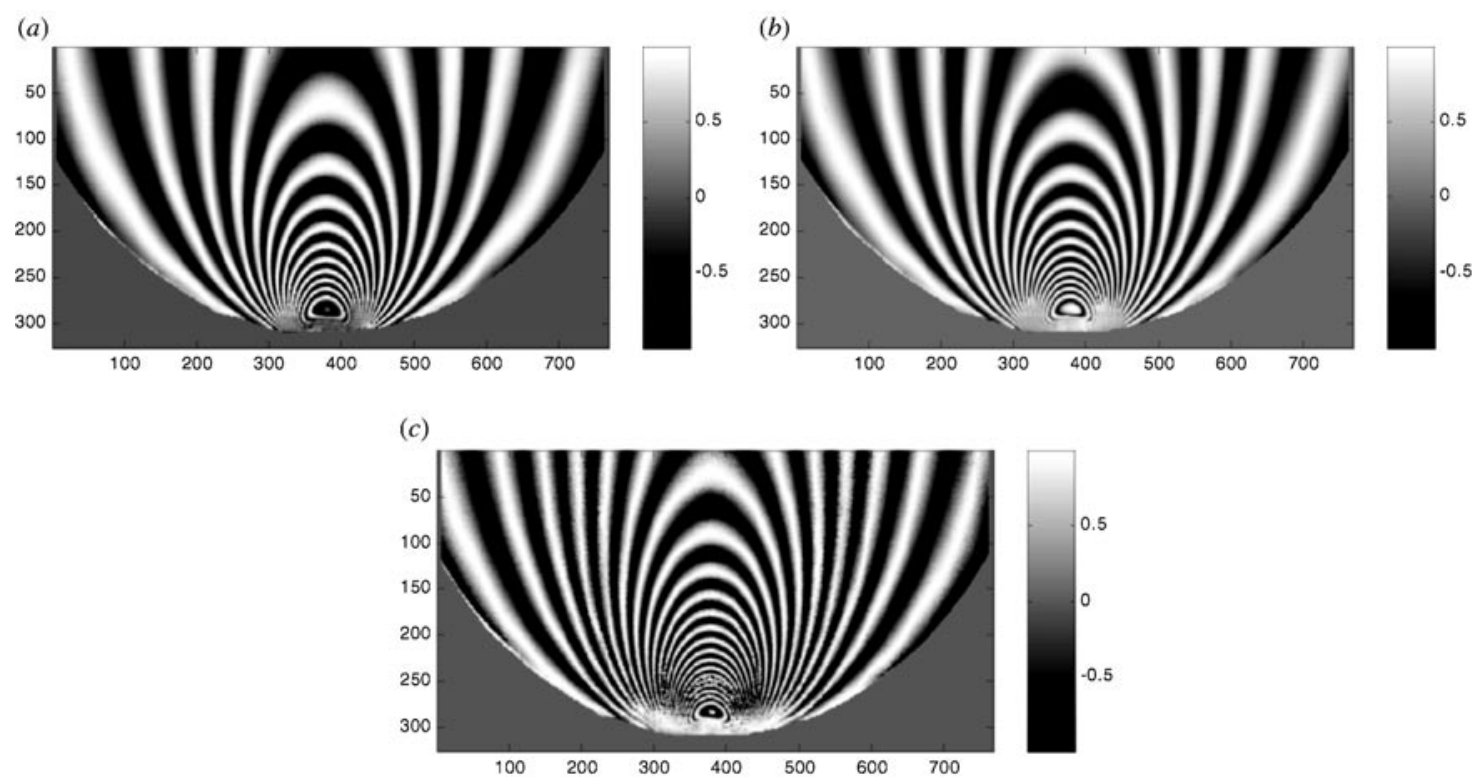

Figure 6. (a) $-(c)$ R, G and B normalized fringe patterns, respectively, generated from the images depicted in figure 1.

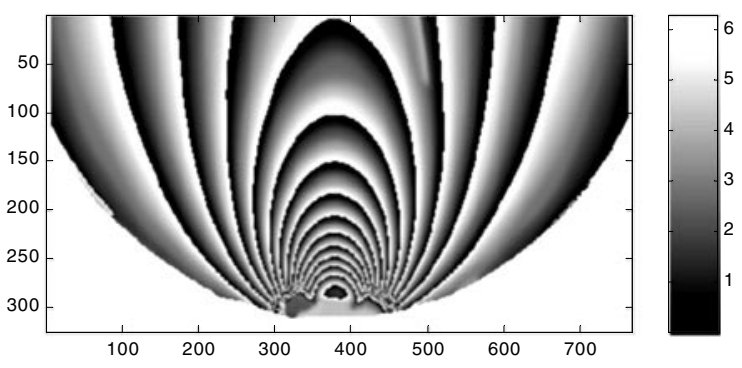

Figure 7. Isochromatic phase $\delta_{\mathrm{R}}$ obtained by the RTP from the images shown in figure 7 using the $K_{i j}$ constants of table 1 . In this case the RTP parameters were $N=9$ pixels and $\beta=1$.

Figures $8(a)$ and $(b)$ show the results for $\delta_{\mathrm{R}}(x, y)$ obtained by the RTP and the PS methods for column 378 and row 200 respectively. As can be seen in the case of the diametrically loaded disc the results obtained by the PS method almost coincide with the RPT.

To test the algorithm with a more complex case we have demodulated the phase of the $\mathrm{R}$ band for a diametrically loaded ring. Figure 9 shows the three RGB bands of the ring for the CDF configuration of the circular polariscope. In this case, the size of the images was $574 \times 768$ pixels. Figure $10(a)$ shows the wrapped result for $\delta_{\mathrm{R}}(x, y)$. In this case the RTP parameters were $N=9$ and $\beta=1$. As a comparison figure $10(b)$ shows the same result obtained by the PS method. Figures 11(a) and (b) show two profiles, for rows 290 and 520 respectively, obtained for $\delta_{\mathrm{R}}(x, y)$ by the RTP and PS methods. In this case the agreement between the PS and RPT techniques is not so good as in the case of the disc, the main reason being the rapid spatial variation of the modulating phase. Currently we are working on the solution to this problem by looking for an adaptive variation of the neighbourhood size. This, we hope, will improve processing of fringe patterns with slow as well as rapid spatial variations of the modulating phase.
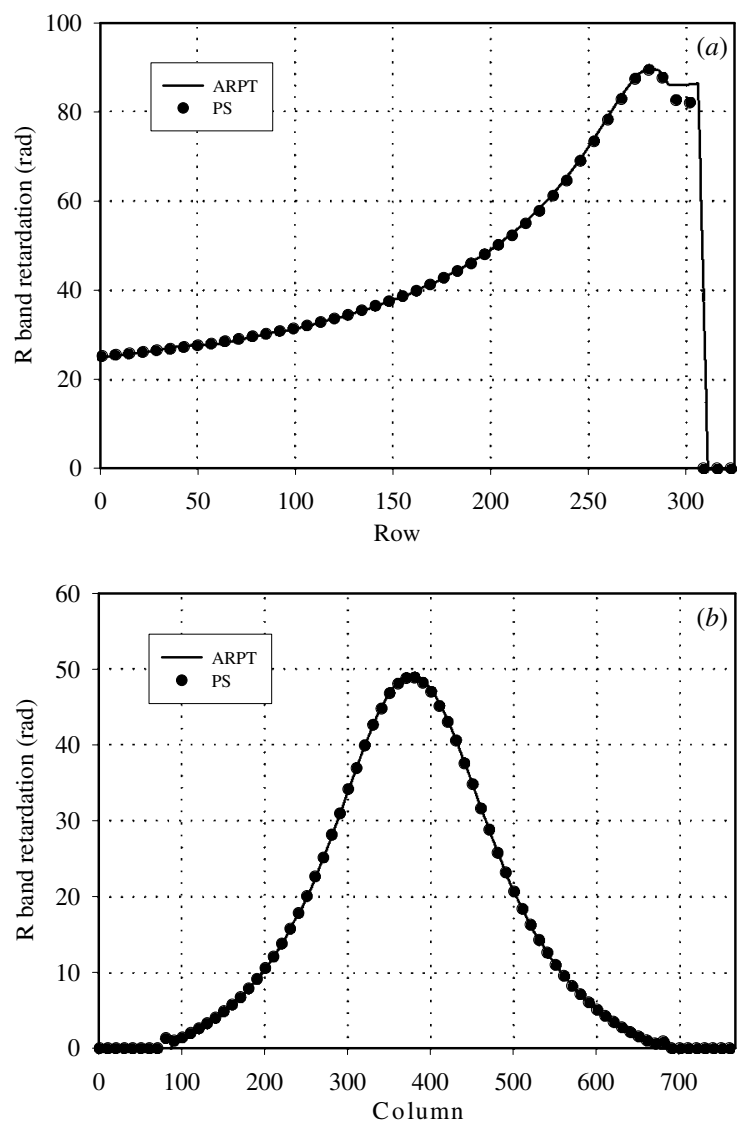

Figure 8. Comparison between the RTP (continuous curve) and the PS (dots) methods, for ( $a$ ) column 378 and $(b)$ row 200, for the disc under diametrical compression.

\section{Acknowledgments}

We wish to thank the European Union, project INDUCE, BRPR-CT97-0805, for financial support of this work. MS and JLM were supported in part by grants from Conacyt, México. 
(a)

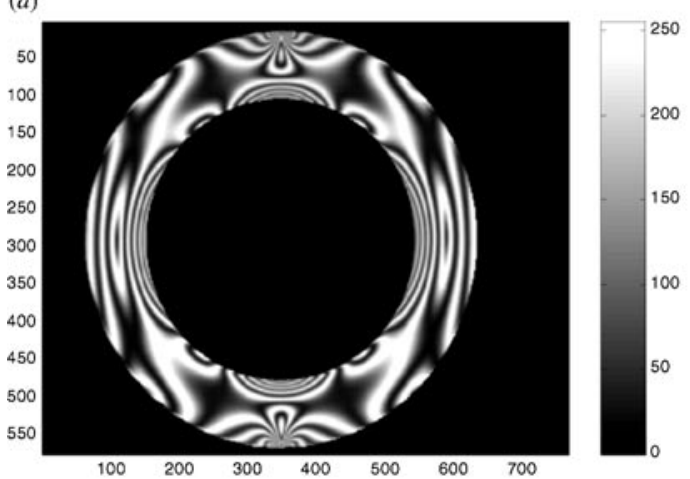

(b)

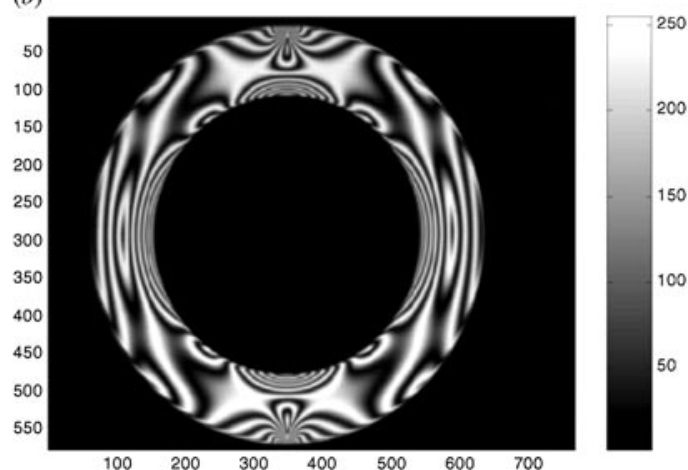

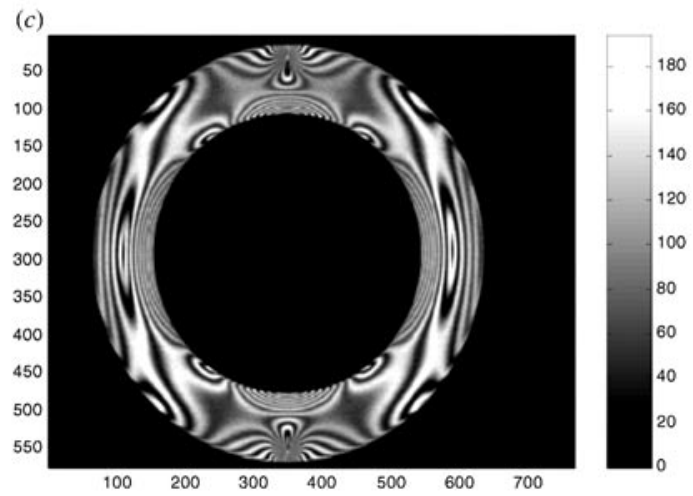

Figure 9. $(a),(b)$ and $(c)$ correspond to the R, G and B bands, respectively, of the isochromatic RGB pattern for a ring under diametrical compression obtained in the $\mathrm{CDF}$ configuration of a circular polariscope.
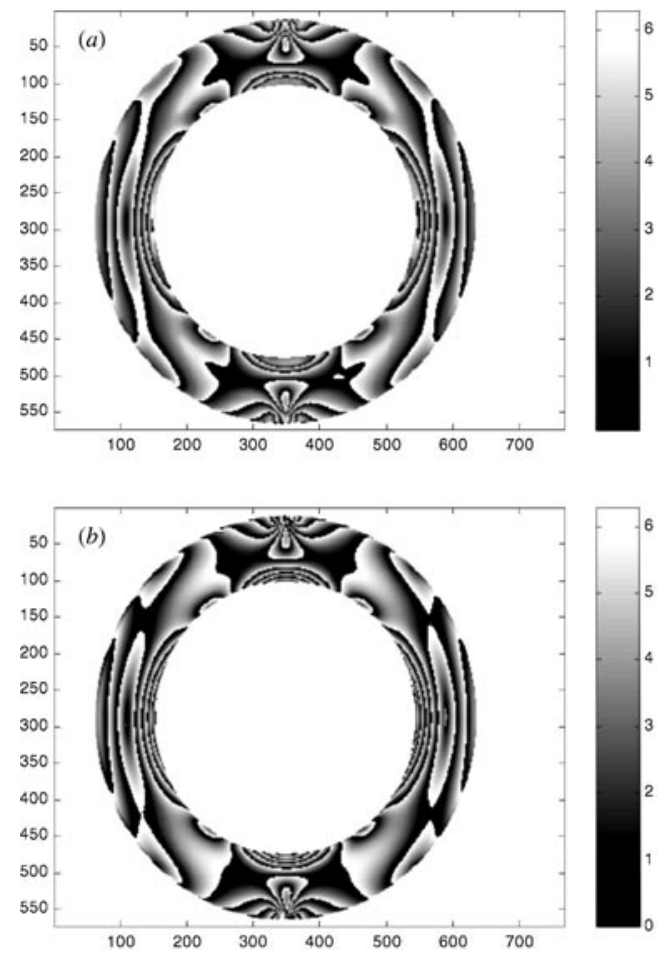

Figure 10. Isochromatic phase $\delta_{\mathrm{R}}$ obtained by (a) RTP method and (b) PS method. In the case of the RTP method the phase is shown wrapped for the sake of clarity and the parameters used were $N=9$ and $\beta=1$.
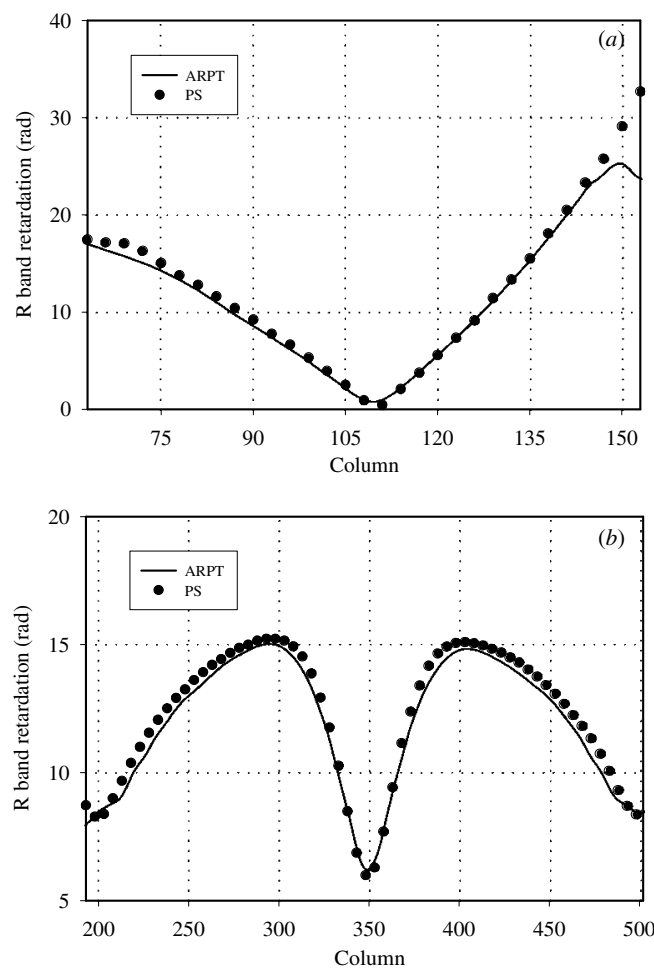

Figure 11. Comparison between the RTP (continuous curve) and the PS (dots) methods, for ( $a$ ) row 290 and $(b)$ row 520, for the ring under diametral compression. 


\section{References}

[1] Buckberry C and Towers D 1995 Automatic analysis of isochromatic and isoclinic fringes in photoelasticity using phase-measuring techniques Meas. Sci. Technol. 6 1227-35

[2] Morimoto Y, Morimoto Y and Hayashi T 1994 Separation of isochromatics and isoclinics using Fourier transform Exp. Tech. September/October 13-17

[3] Carazo-Alvarez J, Haake S J and Patterson E A 1994 Completely automated photoelastic fringe analysis $O p t$. Lasers Eng. 21 133-49

[4] Yoneyama S, Shimizu M, Gotoh J and Takashi M 1998 Photoelastic analysis with a single tricolor image Opt. Lasers Eng. 29 423-35

[5] Ajovalasit A, Barone S and Petrucci G 1995 Towards RGB photoelasticity: full-field automated photoelasticity in white light Exp. Mech. September 193-200

[6] Ekman M J and Nurse A D 1998 Completely automated determination of two-dimensional photoelastic parameters using load stepping Opt. Eng. 37 1845-51

[7] Quiroga J A and Gonzalez-Cano A 2000 Separation of isoclinics and isochromatics from photoelastic data using a regularized phase-tracking technique Appl. Opt. 39 2931-40

[8] Marroquin J L, Rivera M, Botello S, Rodriguez-Vera R and Servin M 1999 Regularization methods for processing fringe-patterns images Appl. Opt. 38 788-94

[9] Servin M and Quiroga J A 2001 Isochromatics demodulation from a single image using a regularized phase tracking technique J. Mod. Opt. 48 521-31
[10] Servin M, Marroquin J L and Cuevas F J 1997 Demodulation of a single interferogram by use of a two-dimensional regularized phase-tracking technique Appl. Opt. 36 4540-8

[11] Ströbel B 1996 Processing of interferometric phase maps as complex-valued phasor images Appl. Opt. 35 2192-8

[12] Villa J, Quiroga J A and Servin M 2000 Improved regularized phase-tracking technique for the processing of squared grating deflectograms Appl. Opt. 39 502-8

[13] Quiroga J A and Gonzalez-Cano A 2000 Separation of isoclinics and isochromatics from photoelastic data using a regularized phase-tracking technique Appl. Opt. 39 2931-40

[14] Servin M, Marroquin J L and Cuevas F J 2001 A robust strategy for demodulation of closed fringe interferograms using a regularized phase tracking system J. Opt. Soc. Am. A 18 689-95

[15] Theocaris P S and Gdoutos E E 1979 Matrix Methods in Photoelasticity (Berlin: Springer)

[16] Quiroga J A and Gonzalez-Cano A 1997 Phase measuring algorithm for extraction of isochromatics of photoelastic fringe patterns Appl. Opt. 36 8397-402

[17] Ng T W, Sajan M R and Asundi A 1997 Photoelastic illumination by using cathode-ray-tube displays Appl. Opt. 36 3601-85

[18] Asundi A and Sajan M R 1995 Multiple LED camera for dynamic photoelasticity Appl. Opt. 34 2236-40

[19] Quiroga J A, Gomez-Pedrero J A and Garcia-Botella A 2001 Algorithm for fringe pattern normalization Opt. Commun. $19743-51$ 